\title{
Spectroscopy, persistent hole burning, and holographic applications of naphtophthalocyanine/haloanthracene systems
}

\author{
R.S. Pandher ${ }^{\mathrm{a}}$, A.A. Gorokhovsky ${ }^{\mathrm{a}, *}$, Z. Liu ${ }^{\mathrm{b}}$, I.V. Solomatin ${ }^{\mathrm{b}}$, Demetri Psaltis ${ }^{\mathrm{b}}$ \\ ${ }^{a}$ Department of Engineering Science and Physics, The College of Staten Island and Graduate School of CUNY, 2800 Victory Blvd., \\ Staten Island, NY 10314, USA \\ ${ }^{\mathrm{b}}$ California Institute of Technology, Pasadena, CA 91125, USA
}

\begin{abstract}
We report on the spectroscopic and hole burning properties of free-base naphtophthalocyanine with and without 1chloranthracene in polymer host. These materials exhibit a strong $0-0$ absorption band in the region $800 \mathrm{~nm}$ matching the wavelength range of most semiconductor and Ti:Sapphire lasers. The materials studied feature two persistent hole burning photochemical mechanisms, i.e. one-photon proton tautomerization, and two-photon-gated hole burning likely due to donor-acceptor electron transfer. The last mechanism includes the long living intermediate state. The hole width at $1.5 \mathrm{~K}$ is about $300-400 \mathrm{MHz}$ depending on the hole burning conditions, while the inhomogeneous width is about $10^{7} \mathrm{MHz}$. Holographic storage is an important potential application of these materials. We demonstrated 10 plane wave holograms angularly multiplexed at one frequency channel in a spectral hole burning medium. It was shown that the $M / \#$ is still a valid system metric and the measured $M / \#$ in one frequency channel is about 0.01 . (C) 2002 Elsevier Science B.V. All rights reserved.
\end{abstract}

Keywords: Spectral hole burning; Naphtophthalocyanines; Photon-gated electron transfer; Holography; $M$ /\#; Angular multiplexing

\section{Introduction}

The donor-acceptor (D-A) electron transfer complexes of porphyrin derivatives and halogenated aromatic molecules have been extensively studied as potential materials for frequency selective optical storage based on persistent spectral hole burning (PSHB). Many of these materials exhibit photon-gated two-photon PSHB mechanisms [1-4], which permit nondestructive

\footnotetext{
*Corresponding author. Tel.: +1-718-982-2815; fax: +1718-982-2830.

E-mail address: gorokhovsky@postbox.csi.cuny.edu (A.A. Gorokhovsky).
}

one-photon readout. In addition, some of these systems have high hole burning efficiency and allow fast single shot hole burning in the nanosecond [1] and even the picosecond [3] time scale. In phthalocyanines, due to the increase in size of their skeleton and $\pi$-electron system, the position of the $\mathrm{S}_{0}-\mathrm{S}_{1}$ transition is shifted to the infrared $(700-800 \mathrm{~nm})$ as compare to 630 $660 \mathrm{~nm}$ in porphyrin derivatives. Hole burning in the spectra of several free-base and metallonaphtophthalocyanines has been studied in [5]. These materials exhibit a strong $0-0$ absorption band in the region $800 \mathrm{~nm}$, matching the wavelength range of most semiconductor and Ti:Sapphire lasers. An important application of PSHB 
media is holographic ultra-high density optical storage. Recently, the recording of 12,000 holograms at a single location by frequency multiplexing has been demonstrated [6]. Combining angle and frequency multiplexing can further increase the storage capacity.

In this paper, we report on comparison of spectroscopic and hole burning properties of two systems, i.e. free-base naphtophthalocyanine, and its mixture with 1-chloranthracene in polyvinyl butyral (PVB) matrix. In the first material, the hole burning mechanism is a one-photon proton tautomerization [7] with quantum efficiency of about $1 \%$ [5]. In the second system, proton tautomerization, as well as two-photon-gated hole burning, were observed. This permitted us to compare the efficiency for both mechanisms, though we cannot expect a high gating ratio in this material. Results on their applications to holography, particularly for angular multiplexing and $M / \#$ measurement, are discussed as well.

\section{Samples}

The guest molecules under investigation were 2,11,20,29-tetra-tert-butyl-2,3-naphthalocya- nine $\left(\mathrm{H}_{2}\right.$-TBNP) and a mixture of $\mathrm{H}_{2}$-TBNP and 1-chloroanthracene (1-ACl). The PVB matrix was used for films with thickness in the range of $100-500 \mu \mathrm{m}$. All chemicals were purchased from Aldrich. The doping concentration was about $3 \times 10^{-5} \mathrm{~mol} / 1$ for $\mathrm{H}_{2}$-TBNP to avoid aggregation, and between $10^{-5}$ and $10^{-4} \mathrm{~mol} / 1$ for $1-\mathrm{ACl}$. Optical samples were prepared by dissolving PVB in dichloromethane; then the required amounts of $\mathrm{H}_{2}$-TBNP and 1- $\mathrm{ACl}$ were added. The mixture was left for evaporation for 7 days in a Petri dish at room temperature.

\section{Spectroscopy and spectral hole burning}

A representative example of absorption spectra of $\mathrm{H}_{2}-\mathrm{TBNP} / 1-\mathrm{ACl}$ in $\mathrm{PVB}$ at $8 \mathrm{~K}$ is shown in Fig. 1. The intense band located around $783 \mathrm{~nm}$ corresponds to the $0-0$ origin of the $\mathrm{S}_{0}-\mathrm{S}_{1}$ transition of $\mathrm{H}_{2}$-TBNP. Vibronic bands are clearly seen at $743 \mathrm{~nm}$ (vibrational energy is $870 \mathrm{~cm}^{-1}$ ) and $698 \mathrm{~nm}\left(1560 \mathrm{~cm}^{-1}\right)$. The narrow peaks located between 400 and $300 \mathrm{~nm}$ belong to 1$\mathrm{ACl}$. The absorption spectrum of $\mathrm{H}_{2}$-TBNP looks similar to that of $\mathrm{H}_{2}-\mathrm{TBNP} / 1-\mathrm{ACl}$ which points to the absence of the spontaneous ground state $\mathrm{D}-\mathrm{A}$

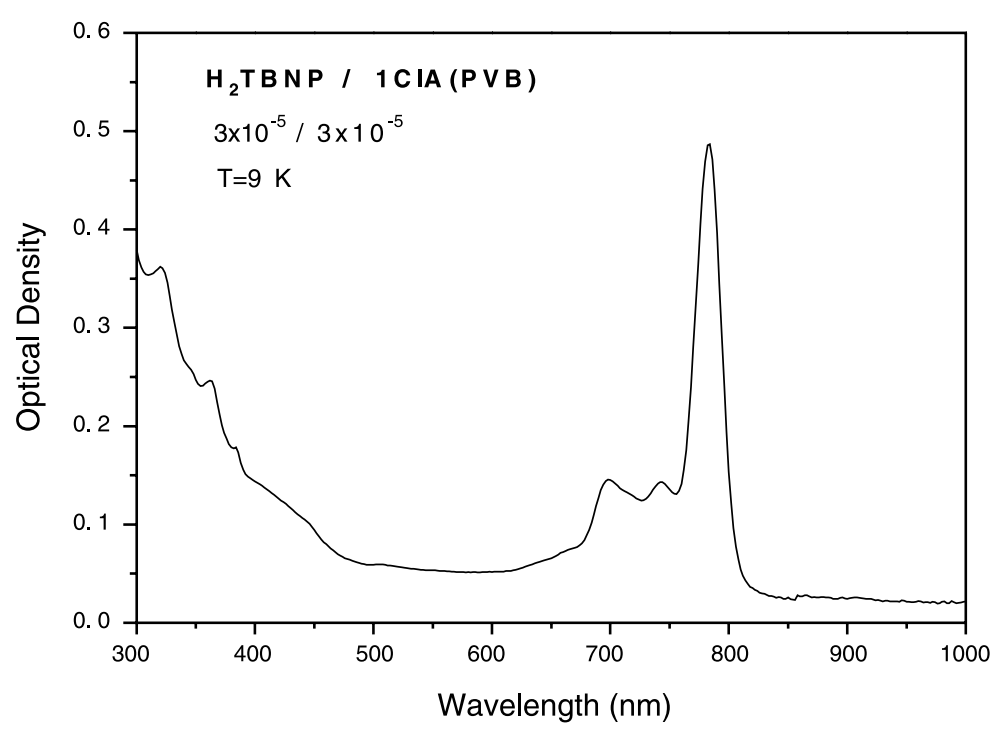

Fig. 1. Low temperature absorption spectrum of $\mathrm{H}_{2}-\mathrm{TBNP} / 1-\mathrm{ACl}$ in $\mathrm{PVB}$. 
complex formation at the used dopant concentrations. At room temperature, the position and width of the $0-0$ band of $\mathrm{H}_{2}-\mathrm{TBNP} / 1-\mathrm{ACl}$ are almost identical to those at $8 \mathrm{~K}$, suggesting a large inhomogeneous broadening of about $10^{7} \mathrm{MHz}$ [5] at low temperatures.

Spectral hole burning measurements were carried out near the maximum of the $0-0$ absorption band at the temperature $1.5 \mathrm{~K}$. A single frequency tunable Ti:Sapphire laser with line width $<1 \mathrm{MHz}$ provided a frequency-selective excitation at $792 \mathrm{~nm}\left(\lambda_{1}\right)$. Hole spectra were detected in transmission following hole burning at an intensity reduced by $2-3$ orders of magnitude to avoid hole profile distortion. The second harmonic of a $\mathrm{CW} \mathrm{Nd}$ laser at $530 \mathrm{~nm}\left(\lambda_{2}\right)$ was used to provide a nonselective gating excitation. The exposure lengths and the time delay between both beams were controlled by two mechanical shutters.

The hole spectra for $\mathrm{H}_{2}-\mathrm{TBNP} / 1-\mathrm{ACl} / \mathrm{PVB}$ are shown in Fig. 2 for one- and two-photon hole burning. Spectrum (a) corresponds to hole burning with a single $50 \mathrm{~ms}$ pulse of the irradiation at $\lambda_{1}$ with intensity $2.1 \mu \mathrm{W} / \mathrm{cm}^{2}$. The relative hole depth

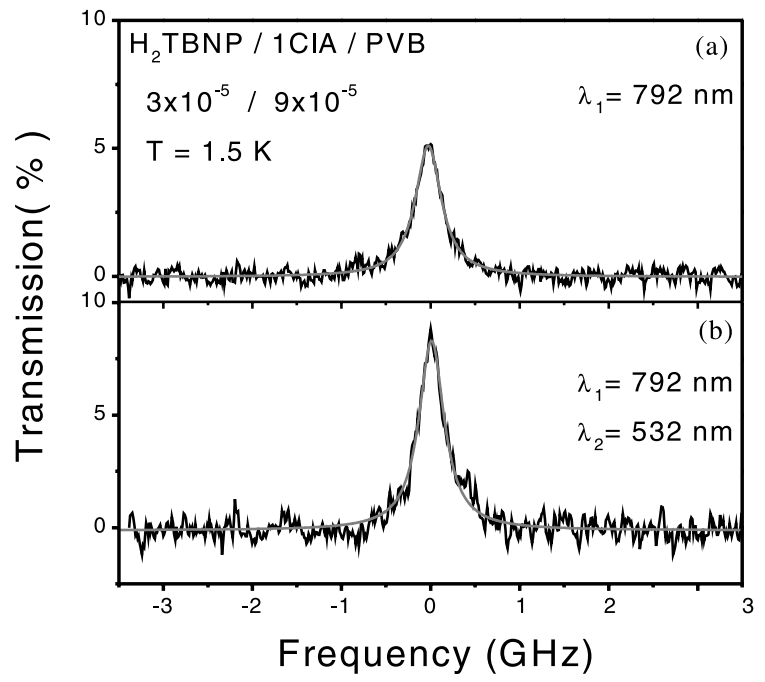

Fig. 2. Hole profiles in transmission spectra of $\mathrm{H}_{2}-\mathrm{TBNP} / 1$ $\mathrm{ACl}$ in PVB at $1.5 \mathrm{~K}$. (a) Hole burning at $\lambda_{1}=792 \mathrm{~nm}$ with a single $50 \mathrm{~ms}$ pulse and the intensity of $2.1 \mu \mathrm{W} / \mathrm{cm}^{2}$. (b) Hole burning with the same irradiation at $\lambda_{1}=792 \mathrm{~nm}$ and additional irradiation at $\lambda_{2}=530 \mathrm{~nm}\left(50 \mathrm{~ms}, 50 \mu \mathrm{W} / \mathrm{cm}^{2}\right)$. Fitting curves are Lorentzians. is about $5 \%$, and the hole width is about $350 \mathrm{MHz}$, which is close to the results for materials without $1-\mathrm{ACl}$ doping at the same conditions. Therefore, we may conclude that the hole burning mechanism is a one-photon proton tautomerization [5]. The hole burning with the same $\lambda_{1}$ illumination, but with the addition of a single $50 \mathrm{~ms}$ pulse of illumination at $\lambda_{2}$ with intensity $50 \mu \mathrm{W} / \mathrm{cm}^{2}$, shows (Fig 2(b)) almost two-fold increase of the hole depth. No visible change in the hole width was observed. This gating amplification depends on the time delay between $\lambda_{1}$ and $\lambda_{2}$ excitation pulses and disappears with increase of delay, with time constant $0.3 \mathrm{~s}$ (see Fig. 3). No gating hole amplification was observed for the materials with 1-ACl concentration $3 \times 10^{-5} \mathrm{~mol} / 1$ or less, or without doping. Consequently, the two-photon irradiation induces a new hole burning mechanism in $\mathrm{H}_{2}$-TBNP- and 1-ACl-doped PVB in addition to proton tautomerization. This mechanism has almost the same overall quantum efficiency of about $1 \%$ [5].

Based on the results of the study of gated hole burning for porpyrins in the presence of halogenated aromatic compounds [1-3], we propose the following two-photon gating mechanism in our case. It involves the selective excitation by the $\lambda_{1}$-photon of $\mathrm{H}_{2}$-TBNP (donor, D); electron transfer reaction in the excited (likely triplet) states of donor to 1-ACl (acceptor, A) through the intermediate state (IS); and the induced $\lambda_{2}$ photon formation of a stable photo-product SP, i.e. $\mathrm{D}+\lambda_{1} \rightarrow \mathrm{D}^{*}+\mathrm{A} \rightarrow \mathrm{IS}+\lambda_{2} \rightarrow \mathrm{SP}$. In the absence of the $\lambda_{2}$-photon, IS decays with time constant $0.3 \mathrm{~s}$ to the ground state of the donor: IS $\rightarrow$ D. In contrast to the results in [1], and in correspondence with the observations in [2], the long living IS is not likely the triplet state of $\mathrm{H}_{2-}$ TBNP, since in general phthalocyanines have a much shorter triplet state lifetime of the order of $1 \mathrm{~ms}$ [8]. An unusual feature of the observed gated holes is their small width of about $400 \mathrm{MHz}$, which is close to that for nongated materials, and about 10-20 times less than typically observed in other gated PSHB materials [1-4]. The reason for such narrow holes is likely related to a relatively low concentration of acceptor molecules 1-ClA of about $10^{-4} \mathrm{~mol} / \mathrm{l}$ due to the lesser influence of 


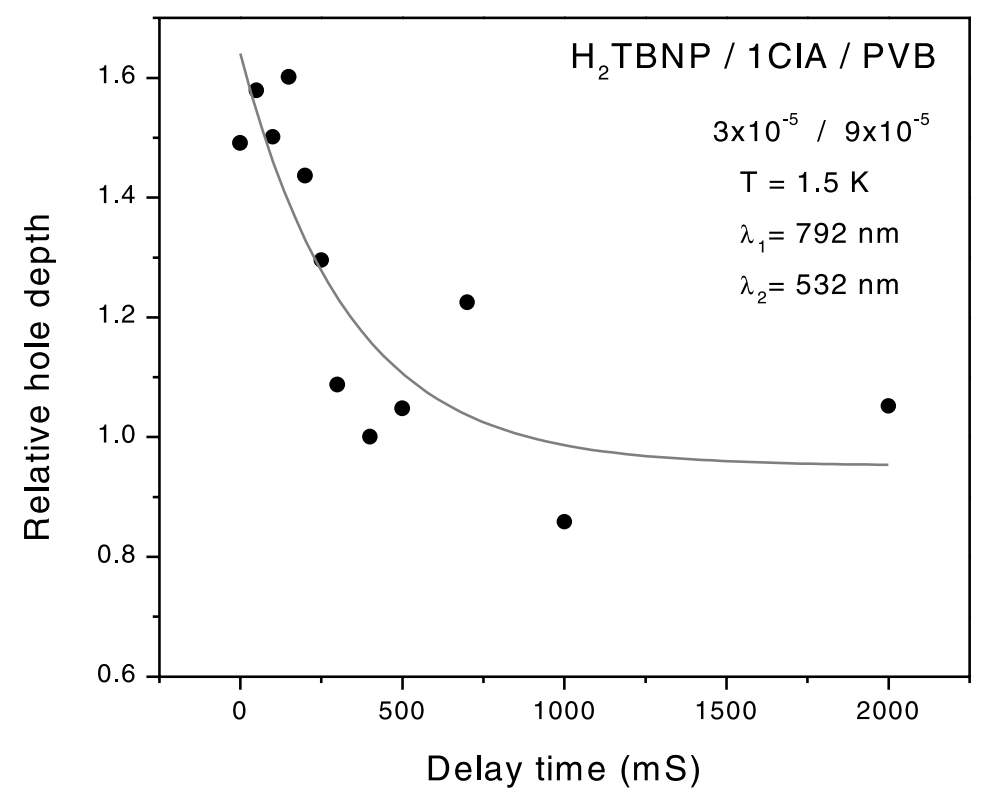

Fig. 3. The gating ratio with respect to the time delay between pulses at $\lambda_{1}=792 \mathrm{~nm}\left(50 \mathrm{~ms}, 2.1 \mu \mathrm{W} / \mathrm{cm}^{2}\right)$ and $\lambda_{2}=530 \mathrm{~nm}(50 \mathrm{~ms}$, $50 \mu \mathrm{W} / \mathrm{cm}^{2}$ ). The fitting curve is an exponential decay with a time constant of $0.33 \mathrm{~s}$.

the electric field induced by $\mathrm{D}-\mathrm{A}$ pair formation [1]. This conclusion requires verification by future experiments on materials with higher acceptor concentration, which are expected to have more efficient gated hole burning.

\section{Measurement of the $M / \#$ in spectral hole burning media}

The frequency domain capability of PSHB media for holographic applications may be improved by angular multiplexing. A system measure of the diffraction efficiency of multiple holograms superimposed in the same volume is called the $M / \#$ [9], and is used extensively with photorefractive crystals and photopolymers. It is defined by $M / \#=M \eta^{1 / 2}$, where $M$ is the number of superimposed holograms and $\eta$ is the equalized diffraction efficiency of each individual hologram. We show that the $M / \#$ is still a valid system metric in spectrally selective media, and we determine the $M / \#$ in one frequency channel.

The experimental setup is shown in Fig. 4. A $\mathrm{CW}$ laser diode at $790 \mathrm{~nm}$ was used. The laser

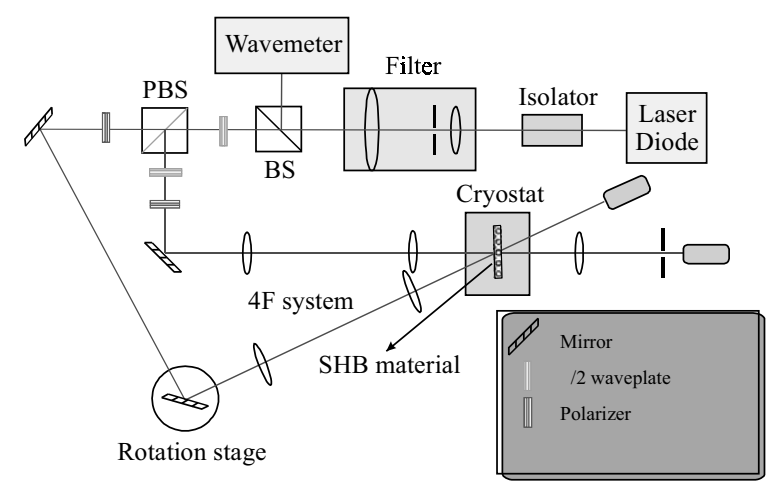

Fig. 4. Experimental holographic setup: angular multiplexing.

beam was split into a signal and a reference beam. The reference beam angle can be changed by rotating the mirror on the rotation stage. The spectral hole burning material was a $0.4 \mathrm{~mm}$ thick film of $\mathrm{H}_{2} \mathrm{TBNP} / \mathrm{PVB}$ with a concentration of $3 \times 10^{-5} \mathrm{~mol} / 1$. The sample was immersed in liquid helium at $2 \mathrm{~K}$.

We recorded a single plane wave hologram where both the reference beam and the signal beam were of intensity $10 \mu \mathrm{W} / \mathrm{cm}^{2}$. A diffraction 
efficiency of $10^{-4}$ was consistently obtained. The optimal recording times were between 5 and $8 \mathrm{~s}$. The angular selectivity curve shown in Fig. 5 was measured by scanning the reference angle after the hologram was recorded. Because of the uniform bleaching during the scanning, the measured central diffraction efficiency is only $0.4 \times 10^{-4}$. During the experiment, an iris filter was used at the back focal plane of the Fourier lens behind the sample (see Fig. 4). This serves two purposes: (i) reduce the scattering and background noise, and (ii) further reduce the selectivity. For thin materials, if the reference beam angle is slightly changed, the reconstructed signal beam angle is changed accordingly and can get partially blocked by the iris filter. The filter gives the combined effect of both angular and peristrophic selectivity. A selectivity of about $0.2^{\circ}$ is obtained, which is consistent with the iris diameter of about $1 \mathrm{~mm}$ and the Fourier lens focal length of about $24 \mathrm{~cm}$.

We angularly multiplexed three, five, seven and ten plane wave holograms. An equal time (1-2s) exposure schedule was used. After the recording, we scanned the reference angle to read out the hologram. The comb functions of ten holograms are shown in Fig. 6. Because holograms can be erased by uniform bleaching during readout, a coarse scan was used. The average diffraction efficiency was about $10^{-6}$. The $M / \#$ obtained from multiplexing three, five, seven and ten holograms is almost the same, and this shows that

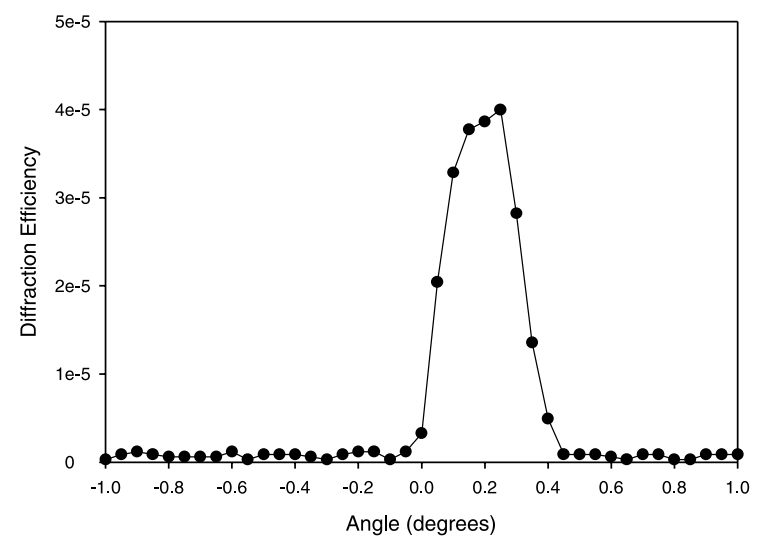

Fig. 5. Angular selectivity. The measured selectivity is about $0.2^{\circ}$.

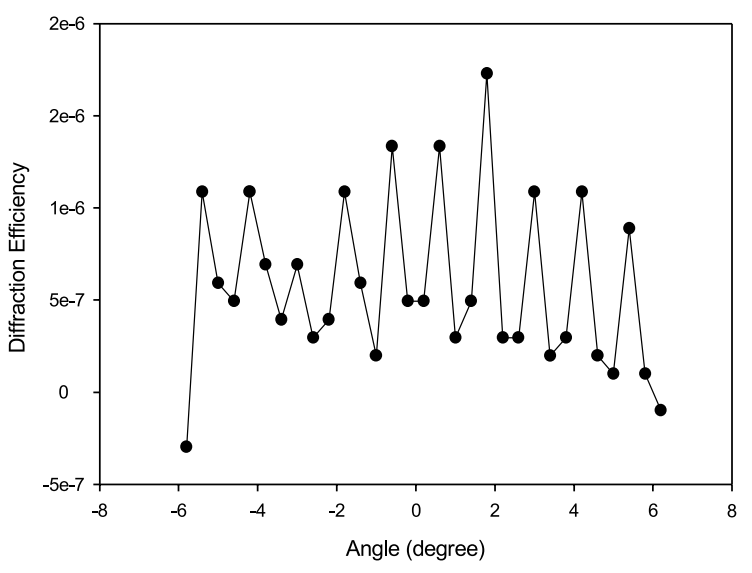

Fig. 6. Ten angularly multiplexed plane wave holograms in $\mathrm{H}_{2}$ TBNP in PVB at $790 \mathrm{~nm}$. The average diffraction efficiency is about $10^{-6}$ which corresponds to a $M / \#$ of 0.01 .

the $M / \#$ is still a good system metric. The measured $M$ /\# was about 0.01 .

\section{Conclusions}

Spectroscopic and hole burning properties of $\mathrm{H}_{2}-\mathrm{TBNP} / 1-\mathrm{ACl}$ in PVB host were studied and compared to that of $\mathrm{H}_{2}$-TBNP in PVB. The absorption spectrum of both materials looks similar in studied concentration range. The $\mathrm{H}_{2}$-TBNP/1-ACl featured two persistent hole burning photochemical mechanisms, i.e. onephoton proton tautomerization common for $\mathrm{H}_{2}$-TBNP, and two-photon-gated hole burning likely due to donor-acceptor electron transfer. The quantum efficiency for both mechanisms was found to be about $1 \%$ at $1-\mathrm{ACl}$ concentration of $10^{-4} \mathrm{~mol} / \mathrm{l}$. The second mechanism includes the IS with lifetime of $0.3 \mathrm{~s}$. The hole width at $1.5 \mathrm{~K}$ is about $300-400 \mathrm{MHz}$, which is close to the results for $\mathrm{H}_{2}$-TBNP at the same conditions, while the inhomogeneous width is about $10^{7} \mathrm{MHz}$. Ten angularly multiplexed plane wave holograms were recorded in $\mathrm{H}_{2}-\mathrm{TBNP} / \mathrm{PVB}$. It was shown that the $M / \#$ is still a valid system metric for PSHB holography, and the measured $M / \#$ in one frequency channel was about 0.01 . The results can be extended to other frequency channels as well. 


\section{Acknowledgements}

The work was supported by the US Air Force Office of Scientific Research under contract F 49620-99-1-0192.

\section{References}

[1] T.P. Carter, C. Brauchle, V.Y. Lee, M. Manavi, W.E. Moerner, J. Phys. Chem. 91 (1987) 3998.
[2] H. Suzuki, T. Shimada, H. Hiratsuka, Appl. Phys. Lett. 70 (1991) 4671.

[3] H. Suzuki, Adv. Mater. 5 (1993) 216 and references therein. [4] I. Renge, U.P. Wild, J. Phys. Chem. A 101 (1997) 4900.

[5] A.V. Turukhin, A.A. Gorokhovsky, C. Moser, I.V. Solomatin, D. Psaltis, J. Lumin. 86 (2000) 399.

[6] B. Plagemann, F.R. Graf, S.B. Altner, A. Renn, U.P. Wild, Appl. Phys. B 66 (1998) 67.

[7] A.A. Gorokhovskii, R.K. Kaarli, L.A. Rebane, JETP Lett. 20 (1974) 216.

[8] R. Avarmaa, Chem. Phys. Lett 46 (1977) 279.

[9] F.H. Mok, G.W. Burr, D. Psaltis, Opt. Lett. 21 (1996) 896. 